\title{
Polar di-halogen molecules solvated in bosonic helium clusters: The paradigm of $\operatorname{ICl}(X)$
}

\author{
M. P. de Lara-Castells, R. Prosmiti, G. Delgado-Barrio, D. López-Durán, and P. Villarreal* \\ Instituto de Matemáticas y Física Fundamental (C.S.I.C.), Serrano 123, E-28006 Madrid, Spain \\ F. A. Gianturco \\ Department of Chemistry and INFM, University of Rome La Sapienza, Piazzale A. Moro 5, 00185 Rome, Italy \\ J. Jellinek \\ Chemistry Division, Argonne National Laboratory, Argonne, Illinois 60439, USA
}

(Received 1 June 2006; published 28 November 2006)

\begin{abstract}
Energies and structures of ${ }^{4} \mathrm{He}_{N^{-}} \mathrm{ICl}(X)$ complexes, $N \leqslant 30$, are determined within the framework of a recently developed Hartree-like approach [Phys. Rev. A 71, 033203 (2005)], in which the He atoms play the role of the electrons and the $\mathrm{I}$ and $\mathrm{Cl}$ atoms play the role of the nuclei. The potential energy of the system is represented as a sum of the $\mathrm{He}-\mathrm{ICl}$ triatomic and $\mathrm{He}-\mathrm{He}$ pair interactions fitted to results of ab initio calculations. The validity of the approach is evaluated through comparisons with the results of "exact" variational calculations performed for the cases of $N=1$ and 2. The procedure, which furnishes also the wavefunctions, allows for simulation of the infrared spectra of the ICl molecule embedded in the bath of $\mathrm{He}_{N}$ clusters. Similarly to the case of the "bare" ICl, when the clusters are formed from bosonic He atoms the absorption selection rules lead only to the $P$ and $R$ branches of the spectra (the $Q$ branches are missing). The spectra exhibit a monotonically increasing blueshift, albeit with a decreasing rate, as the cluster size increases.
\end{abstract}

DOI: 10.1103/PhysRevA.74.053201

\section{INTRODUCTION}

Helium nanodroplets have proven to be a unique matrix in which to conduct high-resolution molecular spectroscopy as well as for synthesizing new molecular species and clusters [1]. Following the pioneering work on the infrared spectra of $\mathrm{SF}_{6}[2,3]$ and later of OCS $[4,5]$ embedded in He droplets, rotationally resolved spectra have been reported for a wide range of systems (see, e.g., Refs. [6-8]). In order to study further the nature of the helium environment and its interaction with dopant molecules on the microscopic scale, highresolution infrared and microwave measurements on small doped helium clusters have been recently performed (see, e.g., Refs. $[9,10])$. Theoretical approaches to describe the structure and ground-state energetics of helium clusters [11] have been provided by zero temperature diffusion Monte Carlo (DMC) [12] and finite temperature Feymann's pathintegral Monte Carlo methods (see, e.g., Ref. [13]). The projection imaginary time spectral evolution implementation of the DMC method by Blume et al. [14] allows for evaluation of the energies of low-lying excited states (see, e.g., Ref. [15]). The recently developed reptation quantum Monte Carlo approach, [16] in which the correlation function is computed explicitly and is fitted to a multi-exponential function, yields excitation energies and spectral weights necessary for simulation of spectra of solvated species (see, e.g., Ref. [13]). The principal difficulty of these methods is the use of the inverse Laplace transform of the time correlation function which is an ill-defined problem [14].

Recently, we have shown that an alternative quantum chemistry-like approach can be used efficiently to obtain en-

\footnotetext{
*Author to whom correspondence should be addressed. Email address: p.villarreal@imaff.cfmac.csic.es
}

PACS number(s): 36.40.Mr, 31.15.Ar, 33.20.Fb, 33.80.Gj

ergies and structural properties of $\mathrm{He}_{N}-\mathrm{Br}_{2}(X)$ complexes [17-19]. The treatment also supplies the system's wave functions, allowing one to carry out spectral simulations. We have demonstrated this for vibrotational Raman spectra of nonpolar diatomic molecules $[17,18]$. Our results point to the central role of the nuclear spin statistics of the surrounding $\mathrm{He}$ atoms in defining the very different spectral features observed experimentally for OCS [4] and $\mathrm{SF}_{6}$ [1] molecules dependently on whether they are solvated in a fermionic or bosonic environment. Using $\mathrm{Br}_{2}(X)$ as a dopant and $\mathrm{He}_{2}$ as a solvent we have shown [20] that a model potential energy surface (PES) of the entire system can be constructed as a sum of ab initio $\mathrm{He}-\mathrm{Br}_{2}$ triatomic interactions [21] and semiempirical $\mathrm{He}-\mathrm{He}$ [22] pair interactions. DMC calculations [23] have indicated that the energies and density distributions for clusters with $N \geqslant 16$ are only weakly dependent on the $\mathrm{He}-\mathrm{Br}_{2}$ interaction, irrespective of whether it is described by the $a b$ initio triatomic potential with minima at T-shaped and linear configurations [21,24], or by a sum of $\mathrm{He}-\mathrm{Br}$ and $\mathrm{Br}-\mathrm{Br}$ Morse potentials with a minimum only at the T-shaped configuration [25].

In this paper, we study the case of ${ }^{4} \mathrm{He}_{N^{-}} \mathrm{ICl}$ clusters, which are different from ${ }^{4} \mathrm{He}_{N^{-}}-\mathrm{Br}_{2}$ systems at least in two important respects. First, ab initio calculations on the triatomic $\mathrm{He}-\mathrm{ICl}$ [26] predict three PES minima, which correspond to "linear" ( $\mathrm{He}$ on the side of the I end of the $\mathrm{ICl}$ molecule), near T-shaped, and "antilinear" (He on the side of the $\mathrm{Cl}$ end of the $\mathrm{ICl}$ molecule) equilibrium structures. Modeling of the PES as a sum of the He- $\mathrm{ICl}$ triatomic and $\mathrm{He}-\mathrm{He}$ pair interactions has been verified by comparison with $a b$ initio computations on $\mathrm{He}_{2}-\mathrm{ICl}$ [27]. The binding energies of the three equilibrium structures decrease from linear to T-shaped to antilinear. This higher, as compared to the case of $\mathrm{He}-\mathrm{Br}_{2}$, degree of anisotropy has a persistent effect even 
in larger $\mathrm{He}_{N^{-}} \mathrm{ICl}$ clusters (we have considered here values of $N$ up to 30 ). Second, $\operatorname{ICl}(X)$ has a permanent dipole moment and therefore simulation studies of its IR spectra bare more relevance to experimental IR spectroscopy of "linear" molecules such as OCS [4]. We shall show that our treatment explains the absence of the $Q$ branches in the spectra of polar molecules embedded in bosonic environments and anticipate their presence in the case of fermionic embedding environments.

The paper is organized as follows. In the next section we present, in the framework of an adiabatic treatment, a brief outline of the Hartree approach, which includes a slight modification of the He-He interaction potential, and describe the theoretical methodology for simulation of infrared (IR) spectra of polar diatomic dopants. In Sec. III, we characterize the model potential energy surface we use, which is based on $a b$ initio calculations on tri- and tetra-atomic clusters $[26,27]$, and present the results obtained. These include cluster energies and density distributions, as well as angular momenta and IR spectra, all considered as a function of cluster size. The results are validated through comparisons with "exact" variational calculations performed for the cases of $N$ $=1$ and 2 . The analysis focuses on the effects of the embedding environment on the IR spectra of the ICl molecule. The summary and the outlook are outlined in Sec. IV.

\section{THEORETICAL TREATMENT}

\section{A. Adiabatic model}

Using satellite coordinates $\left\{\left(\mathbf{r}, \mathbf{R}_{k}\right)\right\}$, where $\mathbf{r}$ is the vector joining the I and $\mathrm{Cl}$ halogen atoms and $\mathbf{R}_{k}$ are the vectors from the center of mass of the ICl molecule to the different $\mathrm{He}$ atoms, one can write the Hamiltonian of the $\mathrm{He}_{N}-\mathrm{ICl}$ system as

$$
\begin{aligned}
H= & -\frac{\hbar^{2}}{2 m} \frac{\partial^{2}}{\partial r^{2}}+U(r)+\frac{\mathbf{j}^{2}}{2 m r^{2}}+\sum_{k=1}^{N} h_{k}\left(\mathbf{R}_{k}, r\right) \\
& +\sum_{k<l} \tilde{V}_{k l}\left(\mathbf{R}_{k}, \mathbf{R}_{l}, \nabla_{k}, \nabla_{l}\right) .
\end{aligned}
$$

It consists [19] of a diatomic part that corresponds to the ICl molecule (the first three terms), $N \mathrm{He}-\mathrm{ICl}$ triatomic Hamiltonians (the fourth term), and all the $\mathrm{He}-\mathrm{He}$ interactions (the fifth term) including not only potential terms but also dynamical couplings [28]. In Eq. (1), $m$ is the reduced mass of the diatomic molecule, $\mathbf{j}$ is the angular momentum associated with $\mathbf{r}$, and $U$ represents the intramolecular diatomic potential. The triatomic Hamiltonians have the form

$$
h_{k}\left(\mathbf{R}_{k}, r\right)=-\frac{\hbar^{2}}{2 \mu} \frac{\partial^{2}}{\partial R_{k}^{2}}+\frac{\mathbf{l}_{k}^{2}}{2 \mu R_{k}^{2}}+W\left(R_{k}, r, \theta_{k}\right),
$$

where $\mu$ is the reduced mass of the $\mathrm{He}-\mathrm{ICl}$ system, $\mathbf{l}_{k}$ is the angular momentum associated with $\mathbf{R}_{k}$, and $W$ represents the atom-diatom intermolecular potential that depends on the pair of $\left(R_{k}, r\right)$ distances and the angle $\theta_{k}$ between the $\mathbf{R}_{k}$ and $\mathbf{r}$ vectors.

We choose a body-fixed (BF) coordinate system with the $Z$ axis parallel to $\mathbf{r}$, and solve the Schrödinger equation

$$
\left[\sum_{k=1}^{R} h_{k}+\sum_{k<l} \tilde{V}_{k l}-E_{\Lambda}^{(N)}(r)\right] \Phi_{\Lambda}^{(N)}\left(\left\{\mathbf{R}_{k}\right\} ; r\right)=0
$$

within the adiabatic approximation (i.e., for different fixed values of the diatomic bond length $r$ ). This can be accomplished by using, for example, the well-known Hartree method. In Eq. (3), each $r$-dependent eigenenergy, labeled by $\Lambda$, the projection of the total orbital angular momentum $\mathbf{L}$ $=\sum_{k=1}^{N} \mathbf{l}_{k}$ on the molecular axis, constitutes an additional potential energy term for the diatomic molecule. As shown below, in a bosonic helium environment the $r$-dependent eigenenergies correspond to the case of $\Lambda=0$, that is to $\Sigma$ states. For a total angular momentum $\mathbf{J}=\mathbf{j}+\mathbf{L}$, with a projection $\Omega=\Lambda$ onto the BF $Z$ axis, and neglecting the Coriolis couplings, the states of the solvated diatomic molecule are described by the Schrödinger equation

$$
\begin{gathered}
\left\{-\frac{\hbar^{2}}{2 m} \frac{\partial^{2}}{\partial r^{2}}+U(r)+E_{\Lambda}^{(N)}(r)+\frac{\hbar^{2}}{2 m r^{2}}\left[J(J+1)+\left\langle\mathbf{L}^{2}\right\rangle\right]\right. \\
\left.-\varepsilon_{j \Lambda \nu}\right\} \chi_{J \Lambda \nu}^{(N)}(r)=0,
\end{gathered}
$$

where $v$ is the stretching (vibrational) quantum number. Since the total orbital angular momentum $\mathbf{L}$ is not a good quantum number, we use as a characteristic quantity the value of $\left\langle\mathbf{L}^{2}\right\rangle$ computed as an average over the distribution of $\mathrm{L}$ values [19]. Thus, the total wave function is approximated as

$$
\Psi^{(N)} \approx \mathcal{D}_{M \Omega}^{J^{*}}\left(\varphi_{r}, \theta_{r}, 0\right) \Phi_{\Lambda}^{(N)}\left(\left\{\mathbf{R}_{k}\right\} ; r\right) \chi_{J \Lambda v}^{(N)}(r),
$$

where $\mathcal{D}_{M \Omega}^{J}$ are Wigner rotation matrices that depend on the polar components of $\mathbf{r}$ in the space-fixed (SF) frame.

\section{B. Overview of the Hartree approach}

In order to obtain the energies and the helium density distributions we use a Hartree-like approach as described in Ref. [19]. Briefly, the wave function of the $N$ bound helium atoms is constructed as a symmetrized Hartree product of single-particle wave functions. For a generic case, when $N_{i}$ bosons occupy the same single-particle orbital labeled as $i$, the $N$-boson total wave function is expressed as

$$
\Phi_{\left(N_{1}, \ldots, N_{M}\right)}^{(N)}=\frac{1}{\sqrt{\mathcal{N}}} \hat{\mathcal{S}}\left(\prod_{i=1}^{N_{1}} \psi_{1}\left(\mathbf{R}_{i} ; r\right) \prod_{i=N_{1}+1}^{N_{1}+N_{2}} \psi_{2}\left(\mathbf{R}_{i} ; r\right) \cdots \prod_{i=\left(N_{1}+\cdots+N_{M-1}\right)+1}^{N} \psi_{M}\left(\mathbf{R}_{i} ; r\right)\right),
$$


where $\sum_{i}^{M} N_{i}=N, M \leqslant N, \hat{\mathcal{S}}$ is the symmetrization operator, and $1 / \sqrt{\mathcal{N}}$ is the normalization factor, in which $\mathcal{N}$ denotes the number of different Hartree products that can be obtained by interchanging bosonic particles occupying different orbitals. As already discussed [19], the total energy can be written in terms of single particle energies of the bosons occupying the $\psi_{i}$ orbitals and the equivalent to Coulomb and exchange integrals appearing in electronic structure theory.

The single-particle wave functions in Eq. (6) are expanded using a finite basis set composed of products of radial and angular functions

$$
\psi_{i}(\mathbf{R} ; r)=\sum_{n l m} C_{n l m}^{i} G_{n}(R ; r) Y_{\ell m}(\theta, \phi),
$$

where $Y_{\ell m}(\theta, \phi)$ are spherical harmonics. The radial basis functions, $G_{n}(R ; r)$, are obtained from the solutions, seeking for the lowest level, of the Schrödinger equation that corresponds to the $\mathrm{He}-\mathrm{ICl}$ triatomic subsystem with a fixed value of $\theta_{n}$,

$$
\left[-\frac{\hbar^{2}}{2 \mu} \frac{\partial^{2}}{\partial R^{2}}+W\left(R ; r ; \theta_{n}\right)-E_{n}(r)\right] g_{n}\left(R ; r ; \theta_{n}\right)=0 .
$$

The calculations were performed for $n_{\max }$ different equidistant values of $\theta_{n}$ in the range $[0, \pi]$. The radial basis used in Eq. (7) is finally obtained from the radial functions $g_{n}\left(R ; r ; \theta_{n}\right)$ through Schmidt orthogonalization. The coefficients, $C_{n l m}^{i}$, are computed using a direct minimization procedure to force convergence to the global minimum [29].

\section{Infrared spectra}

A molecule as $\mathrm{ICl}(X)$ that has a permanent dipole moment in the ground electronic state can be promoted from an initial vibrotational state $|i\rangle$ to a final vibrotational state $|f\rangle$ within the same electronic state by absorption of one photon of frequency $\omega_{i f}$ that matches the energy difference between the initial and final states. This difference belongs to the infrared region. When such a molecule is embedded in a bath of $\mathrm{He}$ atoms, we can envisage a similar process:

$$
\mathrm{He}_{N}-\mathrm{ICl}(i)+\hbar \omega_{i f} \rightarrow \mathrm{He}_{N}-\mathrm{ICl}(f),
$$

where now the indices $i$ and $f$ refer, respectively, to the initial and final states of the entire $\mathrm{He}_{N^{-}} \mathrm{ICl}$ cluster. In the electric dipole approximation within the first order perturbation theory, the absorption intensity is proportional to the square modulus of the matrix element of the transition moment operator, $\boldsymbol{\mu} \cdot \hat{\mathbf{e}}$, computed using the functions defined by Eq. (5); here, $\boldsymbol{\mu}$ is the dipole moment of the solvated molecule, and $\hat{\mathbf{e}}$ defines the polarization of the electric field. Usually, $\boldsymbol{\mu}$ is expressed in a BF reference frame whereas the electric field defines a natural SF frame. Performing a rotation of the BF frame into the SF frame, $\boldsymbol{\mu}^{\mathrm{SF}}=\mathbf{R}^{-1} \boldsymbol{\mu}^{\mathrm{BF}}$, one obtains

$$
\boldsymbol{\mu} \cdot \hat{\mathbf{e}}=\sum_{p, q}(-1)^{p} e_{-p} \mathcal{D}_{p q}^{1^{*}}\left(\varphi_{r}, \theta_{r}, 0\right) \mu_{q}^{\mathrm{BF}},
$$

and the matrix elements of the transition moment become

$$
\begin{aligned}
\left\langle\psi_{i}^{(N)}|\boldsymbol{\mu} \cdot \hat{\mathbf{e}}| \Psi_{f}^{(N)}\right\rangle \propto & \sum_{p, q}(-1)^{p} e_{-p}\left\langle\chi_{f}^{(N)}\left|\mu_{q}\right| \chi_{i}^{(N)}\right\rangle \delta_{\Lambda_{f} \Lambda_{i}} \\
& \times \int d \tau \mathcal{D}_{M_{f} \Omega_{f}}^{J_{f}} \mathcal{D}_{p q}^{1^{*}} \mathcal{D}_{M_{i} \Omega_{i}^{*}}^{J_{i}^{*}} .
\end{aligned}
$$

As ab initio calculations reveal, the dipole moment of the solvated molecule is only weakly affected by the interactions with the surrounding $\mathrm{He}$ atoms and its direction remains along the $\mathrm{ICl}$ bond, and only the $q=0$ component contributes giving rise to a parallel transition. If one considers linearly polarized light and uses the direction of polarization to define the SF $Z$ axis $(p=0)$, one arrives at

$$
\begin{aligned}
\left\langle\Psi_{i}^{(N)}|\boldsymbol{\mu} \cdot \hat{\mathbf{e}}| \Psi_{f}^{(N)}\right\rangle \propto & (-1)^{M_{i}}\left\langle\chi_{f}^{(N)}\left|\mu_{0}\right| \chi_{i}^{(N)}\right\rangle \delta_{\Lambda_{f} \Lambda_{i}}\left(\begin{array}{ccc}
J_{i} & 1 & J_{f} \\
-M_{i} & 0 & M_{i}
\end{array}\right) \\
& \times\left(\begin{array}{ccc}
J_{i} & 1 & J_{f} \\
-\Omega_{i} & 0 & \Omega_{i}
\end{array}\right) .
\end{aligned}
$$

Since in the our case $\Omega=\Lambda$, for $\Sigma$ states the second $3-j$ symbol in Eq. (11) vanishes unless $\Delta J= \pm 1$. Hence, only the $P$ and $R$ branches survive and the $Q$ transitions are not allowed in the spectrum. In contrast, when $\Omega \neq 0$ states are involved (e.g., because of the presence of fermionic components in the solvent and/or the presence of $\Pi, \Delta, \ldots$, states), the $Q$ transitions become allowed as well. A Boltzmann distribution of rotational states of the clusters that corresponds to a given temperature $T$ gives rise to a line of intensity,

$$
\begin{aligned}
I_{f i}^{(N)}(T) \propto & \frac{e^{-\left(\varepsilon_{i} / k T\right)}}{\sum_{i} e^{-\left(\varepsilon_{i} / k T\right)}} \frac{1}{2 J_{i}+1} \sum_{M_{i}} \mid\left\langle\chi_{f}^{(N)}\left|\mu_{0}\right| \chi_{i}^{(N)}\right\rangle\left(\begin{array}{ccc}
J_{i} & 1 & J_{f} \\
-M_{i} & 0 & M_{i}
\end{array}\right) \\
& \times\left.\left(\begin{array}{ccc}
J_{i} & 1 & J_{f} \\
0 & 0 & 0
\end{array}\right)\right|^{2}
\end{aligned}
$$

at a frequency $\omega_{f i}=\left(\varepsilon_{J_{f} 0 v_{f}}^{(N)}-\varepsilon_{J_{i} 0 v_{i}}^{(N)}\right) / \hbar$ [in Eq. (12), the simplified notation $\varepsilon_{i}$ stands for $\left.\varepsilon_{J_{i} 0 v_{i}}^{(N)}\right]$.

A variety of causes may lead to the broadening of the spectral lines. Here we consider the mechanism of vibrational predissociation (VP). An absorption of a photon that leads to a vibrational excitation of the solvated molecule may be followed by energy transfer to bonds involving the $\mathrm{He}$ atoms. These weaker bonds may rupture giving rise to dissociation of the cluster (hence, vibrational predissociation). Following the procedure outlined in Refs. [17,18], one can estimate the width of the line broadening caused by VP. First, one calculates the $\theta$-dependent VP width for the $\mathrm{He}-\mathrm{ICl}\left(v_{f}\right)$ triatomic species, $\gamma_{v_{f} \leftarrow v_{i}}$, using the framework of the adiabatic angular model [30] and generates the angular distribution $\mathcal{D}_{\Lambda}^{(N)}(\theta)$, which is normalized to $N$, by integrating the square modulus of $\Phi_{\Lambda}^{(N)}\left(\left\{\mathbf{R}_{k}\right\} ; r_{e q}\right)$ defined by Eq. (3) over all variables except one. The VP line broadening is then obtained by averaging $\gamma_{v_{f^{\leftarrow} v_{i}}}$ over the angular distribution,

$$
\Gamma_{f i}^{(N)}=\int_{0}^{\pi} d \theta \mathcal{D}_{\Lambda}^{(N)}(\theta) \gamma_{v_{f} \leftarrow v_{i}}(\theta) .
$$


By dressing the lines with Lorentzians of appropriate widths and summing up over all transitions, one arrives at the following expression for the IR photoabsorption cross section

$$
\sigma_{N}(\omega ; T)=\frac{1}{\pi} \sum_{f, i} \frac{\Gamma_{f i}^{(N)}}{\hbar^{2}\left(\omega-\omega_{f i}\right)^{2}+\left(\Gamma_{f i}^{(N)} / 2\right)^{2}} I_{f i}^{(N)}(T) .
$$

The cross section satisfies the condition

$$
\int d \omega \sigma_{N}(\omega ; T)=\sum_{f, i} I_{f i}^{(N)}(T) .
$$

\section{RESULTS}

\section{A. Electronic structure calculations and potential energy surfaces}

For the $\mathrm{He}_{2}-\mathrm{ICl}(X)$ tetraatomic cluster, ab initio calculations [27] show that the interaction energy can be accurately represented as the sum of energies of two $\mathrm{He}-\mathrm{ICl}$ triatomic species and the energy of the He-He interaction. Hence, we extend this description to cluster of larger sizes and use the following expression for the interaction energy:

$$
\begin{aligned}
W_{\mathrm{He}_{N}-\mathrm{ICl}}\left(\left\{\mathbf{R}_{k}\right\}_{k=1}^{N} ; r\right)= & \sum_{k=1}^{N} W_{\mathrm{He}_{k}-\mathrm{ICl}}\left(\mathbf{R}_{k} ; r\right) \\
& +\sum_{k<l} V_{\mathrm{He}_{k}-\mathrm{He}_{l}}\left(\left|\mathbf{R}_{k}-\mathbf{R}_{l}\right|\right) .
\end{aligned}
$$

$A b$ initio calculations of the $\mathrm{He}-\mathrm{ICl}(X)$ interaction have been carried out at the $\operatorname{CCSD}(\mathrm{T})$ level of the theory fixing the $\mathrm{ICl}(X)$ stretch at its equilibrium bond distance $r=r_{e}$ $=2.321 \AA$ (see Ref. [26]). To account for the dependence on $r$, two sets of additional $a b$ initio computations for $\mathrm{He}-\mathrm{ICl}$ using the same methodology have also been performed. The interaction energies are calculated for several intermolecular distances $R$ for $11 \theta$ values between $0^{\circ}$ and $180^{\circ}$ and two $\mathrm{ICl}$ bond lengths of $r_{-}=2.271$ and $r_{+}=2.386 \AA$. These $r$ values correspond to the peaks of the first excited vibrational level of the bare $\operatorname{ICl}(X)$ molecule. The results of the $\operatorname{CCSD}(\mathrm{T})$ calculations of the interaction energies are listed in Table I.

Following the procedure outlined in Ref. [26] the entire PES is described analytically. To this end, we represent the $R$-dependent interaction potential, for fixed values of $r$ and $\theta$, by a Morse-vdW function whose parameters are obtained by a nonlinear least square fit and are listed in Table II (for $r$ $=r_{e}$, see Table III in Ref. [26]). In order to define the PES over a range of $r$ values large enough for characterization of the first two $(v=0$ and 1$)$ vibrational levels of $\operatorname{ICl}(X)$, necessary for the spectral simulations, a quadratic dependence of the energy on $r$ has been assumed. In practice, for each orientation $\theta_{i}(i=1-11)$, the coefficients $A$ and $B$ of the expansion

$$
W\left(R, r ; \theta_{i}\right)=A\left(R, \theta_{i}\right)\left(r-r_{e}\right)^{2}+B\left(R, \theta_{i}\right)\left(r-r_{e}\right)+W\left(R, r_{e}, \theta_{i}\right)
$$

are readily obtained from the corresponding interaction energies $W\left(R, r_{-}, \theta_{i}\right)$ and $W\left(R, r_{+}, \theta_{i}\right)$. The model potential re- produces the $a b$ initio results very well with a maximum standard deviation of $0.5 \mathrm{~cm}^{-1}$ and an average standard deviation of $0.17 \mathrm{~cm}^{-1}$ over the entire range of $r, R$, and $\theta$ values considered (see last column of Table II).

The $\mathrm{He}-\mathrm{ICl}$ potential energy surface exhibits three minima. Over the $r$ values considered, the global minimum with an energy of $-58.625 \mathrm{~cm}^{-1}$ at $R=3.86 \AA$ and $r$ $=2.321 \AA$ corresponds to a linear He-I-Cl $\left(\theta=0^{\circ}\right)$ configuration. The second minimum, with energy of $-40.116 \mathrm{~cm}^{-1}$ at $R=3.80 \AA$ and $r=2.271 \AA$ corresponds to a near T-shaped $\left(\theta=110.9^{\circ}\right)$ configuration of the complex. The third minimum is found for the antilinear He-Cl-I geometry. Its energy is $-38.032 \mathrm{~cm}^{-1}$ and it is located at $R=5.12 \AA$ and $r$ $=2.321 \AA$. The barrier between the linear and the near T-shaped configurations is at $-15.35 \mathrm{~cm}^{-1}$ and it is located at $R \approx 4.44 \AA, \theta \approx 60^{\circ}$, and $r=2.321 \AA$. The characteristics of the barrier between the near T-shaped and the antilinear configurations are $-22.49 \mathrm{~cm}^{-1}, R \approx 4.98 \AA, \theta \approx 141.8^{\circ}$, and $r$ $=2.386 \AA$. The three potential minima and the barriers between them are displayed in Fig. 1. As is clear from the figure, the minimum that corresponds to the near T-shaped configuration is sensitive to $r$-the smaller the value of $r$, the lower the minimum. The other two minima show only negligible variations with $r$. Thus, the energy difference between the minima that correspond to the linear and the near T-shaped configurations increases as the $r$ bond length becomes larger.

For spectral simulations, it is also necessary to estimate the dipole moment $\boldsymbol{\mu}$ of the ICl molecule in the presence of He atoms. $\operatorname{CCSD}(\mathrm{T})$ calculations performed for various configurations of the clusters with $N=1$ and 2 show that the component of the dipole moment along the $\mathrm{I}-\mathrm{Cl}$ bond is one or two orders of magnitude larger than the component orthogonal to it. The magnitude of the dipole moment in the triatomic case is sensitive to the value of $r$, but its dependence on $R$ and $\theta$ is only minor. Similar values are obtained for the tetraatomic system, as could be expected from the nature of the intermolecular interactions, which essentially coincide with the dipole moment of the isolated ICl molecule. Therefore, irrespective of the cluster size, we used the values of 1.140, 1.284, and 1.465 Debye computed for the three distances, $r_{-}, r_{e}$, and $r_{+}$, respectively, and to define $\mu(r)$ as a linear function of $r$.

The $\operatorname{ICl}\left(X^{1} \Sigma^{+}\right)$intramolecular interaction $U(r)$ was approximated by a Morse function [31] with the following values of the parameters: well depth $D=17557.411 \mathrm{~cm}^{-1}$, characteristic inverse length $\alpha=1.8492349 \AA^{-1}$, and equilibrium distance $r_{e}=2.321 \AA$. For the He-He interaction we employed a parametrized potential of Aziz and Slaman [22]. To avoid divergences in the potential at vanishing distances between the He atoms, a truncation of the potential is employed (for details, see Ref. [19]).

\section{B. Helium energies and density distributions}

In the calculations presented here, the following masses (amu) were used: $m_{\mathrm{I}}=126.904473, m_{\mathrm{Cl}}=34.968853$, and $m{ }^{4} \mathrm{He}=4.00260$. A grid of 4096 points in the $R$ range of 2.0-20.0 A was employed to numerically solve Eq. (8), us- 
TABLE I. CCSD $(\mathrm{T})$ interaction energies for the He-ICl molecule obtained with the aug-cc-pV5Z+ $(3 s 3 p 2 d 2 f 1 g)$ basis set for the He and SDB-cc-pVTZ ECP for I at indicated values of $R, r$, and $\theta$. Distances are in $\AA$ and energies in $\mathrm{cm}^{-1}$.

\begin{tabular}{|c|c|c|c|c|c|c|c|c|c|c|c|}
\hline \multicolumn{12}{|c|}{$\Delta E\left(\mathrm{~cm}^{-1}\right)$} \\
\hline$R / \theta$ & $0^{\circ}$ & $22.5^{\circ}$ & $45^{\circ}$ & $67.5^{\circ}$ & $78.75^{\circ}$ & $90^{\circ}$ & $101.25^{\circ}$ & $112.5^{\circ}$ & $135^{\circ}$ & $157.5^{\circ}$ & $180^{\circ}$ \\
\hline \multicolumn{12}{|c|}{$r=2.271 \AA$} \\
\hline 3.00 & 684.11 & & & & & & & & & & \\
\hline 3.25 & 160.24 & & 564.55 & & & 121.03 & 32.48 & 43.40 & & & \\
\hline 3.5 & -13.65 & 99.57 & 202.43 & 136.04 & 76.60 & 20.23 & -21.94 & -22.86 & 714.36 & & \\
\hline 3.75 & -56.09 & 0.22 & 55.87 & 33.99 & 9.84 & -14.38 & -34.45 & -39.34 & & & \\
\hline 4.00 & -55.19 & -27.86 & 1.53 & -3.59 & -12.91 & -22.60 & -32.26 & -37.63 & 72.90 & & \\
\hline 4.25 & -43.62 & -30.62 & -15.25 & -14.73 & -21.44 & & & 1.82 & & 267.24 & 457.70 \\
\hline 4.5 & -31.96 & -25.84 & -17.80 & -15.82 & -16.31 & -17.62 & -20.01 & -23.56 & -20.05 & 57.58 & 99.29 \\
\hline 4.75 & -22.82 & & -15.65 & & -13.37 & -13.70 & & & -23.17 & -10.28 & -11.75 \\
\hline 5.00 & -16.22 & -11.86 & -12.51 & -10.87 & -10.43 & -10.37 & -11.07 & -12.98 & -20.14 & -26.39 & -36.69 \\
\hline 5.5 & & & & & & & & & & & -27.60 \\
\hline 6.00 & -4.58 & -4.49 & -4.14 & -3.66 & -3.48 & -3.40 & -3.55 & -4.10 & -6.77 & -11.48 & -14.60 \\
\hline 7.00 & -1.62 & -1.58 & -1.47 & -1.36 & -1.27 & -1.27 & -1.34 & -1.51 & -2.32 & -3.53 & -4.19 \\
\hline 9.00 & -0.33 & -0.31 & -0.28 & -0.28 & -0.28 & -0.26 & -0.28 & -0.33 & -0.44 & -0.57 & -0.64 \\
\hline 11.00 & -0.09 & -0.07 & -0.07 & & -0.07 & -0.07 & -0.07 & & 0.11 & -0.13 & -0.15 \\
\hline \multicolumn{12}{|c|}{$r=2.386 \AA$} \\
\hline 3.00 & 753.11 & & & & & & & & & & \\
\hline 3.25 & 186.93 & & 587.90 & & & 121.97 & 37.54 & 50.41 & & & \\
\hline 3.5 & -5.15 & 112.33 & 211.04 & 135.71 & 75.55 & 20.82 & -18.98 & -17.99 & 806.01 & & \\
\hline 3.75 & -54.77 & 3.90 & 58.41 & 33.27 & 9.14 & -14.07 & -32.90 & -36.73 & & & \\
\hline 4.00 & -56.22 & -27.62 & 1.82 & -4.25 & -13.30 & -22.49 & -31.50 & -36.43 & 93.52 & & \\
\hline 4.25 & -45.08 & -31.36 & -15.67 & -15.23 & & -21.39 & & -30.33 & 9.93 & 385.92 & 700.02 \\
\hline 4.5 & -33.25 & -26.72 & -18.32 & -16.15 & -16.50 & -17.64 & -19.92 & -23.52 & -17.62 & 100.49 & 182.88 \\
\hline 4.75 & -23.82 & & -16.11 & & -13.50 & -13.72 & & & -23.04 & 2.92 & 12.56 \\
\hline 5.00 & -16.94 & -12.30 & -12.87 & -11.02 & -10.50 & -10.39 & -11.13 & -13.15 & -20.78 & -24.00 & -32.61 \\
\hline 5.5 & & & & & & & & & & & -30.49 \\
\hline 6.00 & -4.78 & -4.67 & -4.25 & -3.70 & -3.51 & -3.44 & -3.62 & -4.19 & -7.19 & -12.70 & -16.50 \\
\hline 7.00 & -1.67 & -1.64 & -1.51 & -1.36 & -1.32 & -1.29 & -1.36 & -1.56 & -2.45 & -3.88 & -4.69 \\
\hline 9.00 & -0.33 & -0.31 & -0.31 & -0.28 & -0.28 & -0.28 & -0.28 & -0.33 & -0.44 & -0.61 & -0.68 \\
\hline 11.00 & -0.09 & -0.09 & & & -0.07 & -0.07 & -0.09 & & -0.11 & -0.15 & -0.15 \\
\hline
\end{tabular}

ing a Numerov procedure. Convergence for the ground state was achieved by using $l_{\max }=19,\left|m_{\max }\right|=1$, and $n_{\max }=7$. The convergence thresholds used for the the total energy and the module of its gradient (with respect to the nonredundant variational parameters [29]) were set to $10^{-6}$ and $10^{-5} \mathrm{~cm}^{-1}$, respectively.

As in the case of $\mathrm{He}_{N}-\mathrm{Br}_{2}$ clusters [19] the lowest energy was found for $\Lambda=0$, a sort of " $\Sigma$ " state of the system. Table III shows the energies of the $\mathrm{He}_{N}$ - $\mathrm{ICl}$ clusters at the equilibrium $\mathrm{ICl}$ bond length and for $N$ bosons equally distributed over $M=1,2$, and 3 single-particle orbitals. As expected for a Bose quantum system, the lowest energy corresponds to all the He atoms occupying the same orbital $(M=1)$ : As is clear from the table, the larger the number of occupied orbitals the higher the total energy of the system. One should also note that the relative energy differences between the various $M$ cases increase with the cluster size, as expected because of the larger number of solvent particles contributing to the total binding energy.

In Fig. 2 we show the values of the total energies, $E_{N}$, and single particle evaporation energies, $\left(E_{N}-E_{N-2}\right) / 2$, as a function of the cluster size $N$. For $N=1$ and $2(J=0)$, the Hartree energy values of 18.38 and $30.33 \mathrm{~cm}^{-1}$ can be compared with the "exact counterparts" of 18.29 and $33.51 \mathrm{~cm}^{-1}$ from Refs. [26,27]. In contrast to the $N=1$ case, the $N=2$ size shows a non-negligible relative error of about $9 \%$. Additional variational calculations within the diatomic rotational decoupling approach give an energy value of $33.47 \mathrm{~cm}^{-1}$, which indicates that the error in the Hartree energy is due to the Hartree approximation itself. We see that both total and single particle evaporation energies change continuously and smoothly, without any sign of shell structuring of the solvent 
TABLE II. Parameters for the analitycal representation of the $\operatorname{He}-\mathrm{ICl}(X)$ interaction potential, see Ref. [26]. Distances are in $\AA$, angles in degrees, while energies and standard deviation are in $\mathrm{cm}^{-1}$. Figures in parentheses are powers of 10 .

\begin{tabular}{|c|c|c|c|c|c|c|}
\hline$\theta$ & $\alpha_{0}$ & $\alpha_{1}$ & $\alpha_{2}$ & $\alpha_{3}$ & $\alpha_{4}$ & $s_{d e v}$ \\
\hline \multicolumn{7}{|c|}{$r=2.271 \AA$} \\
\hline 0 & 0.3385 & 1.7726 & 5.4073 & -17038.6 & $6.63825(06)$ & 0.399 \\
\hline 22.5 & 15374.6 & 1.86307 & 1.4134 & 511867 & $-2.25675(07)$ & 0.471 \\
\hline 45 & 0.55926 & 1.60527 & 5.56862 & 61308 & $3.64064(06)$ & 0.124 \\
\hline 67.5 & 6.4676 & 1.6180 & 4.60974 & 145378 & $-1.31992(06)$ & 0.055 \\
\hline 78.75 & 2.6618 & 1.61824 & 4.8144 & 127807 & -80037.4 & 0.068 \\
\hline 90 & 1.24727 & 1.62011 & 4.95133 & 119689 & 585589 & 0.038 \\
\hline 101.25 & 1.77406 & 1.66522 & 4.69542 & 133954 & 410668 & 0.035 \\
\hline 112.5 & 1.61058 & 1.62273 & 4.85811 & 130072 & $1.24403(06)$ & 0.070 \\
\hline 135 & 1.87481 & 1.57011 & 5.55685 & 3581.42 & $8.66566(06)$ & 0.264 \\
\hline 157.5 & 20.8823 & 1.82036 & 5.09964 & 413184 & $-7.81258(06)$ & 0.062 \\
\hline 180 & 44.0871 & 1.90781 & 4.99438 & 639552 & $-1.88314(07)$ & 0.127 \\
\hline \multicolumn{7}{|c|}{$r=2.386 \AA$} \\
\hline 0 & 1.00887 & 1.75698 & 5.13414 & -21139 & $6.63855(06)$ & 0.422 \\
\hline 22.5 & 4629.81 & 1.85099 & 2.05087 & 504859 & $-2.24958(07)$ & 0.503 \\
\hline 45 & 1.74539 & 1.60586 & 5.21264 & 66045 & $2.83059(06)$ & 0.170 \\
\hline 67 & 4.902 & 1.61867 & 4.71026 & 139030 & -776461 & 0.057 \\
\hline 78.75 & 2.86021 & 1.62301 & 4.78306 & 132648 & -215727 & 0.063 \\
\hline 90 & 2.4398 & 1.61913 & 4.7252 & 126275 & 91174.5 & 0.057 \\
\hline 101.25 & 2.05728 & 1.652 & 4.65743 & 140479 & 202868 & 0.035 \\
\hline 112.5 & 2.56862 & 1.58417 & 4.74817 & 131974 & 974458 & 0.087 \\
\hline 135 & 3.59958 & 1.5342 & 5.42394 & -22802.4 & $8.69692(06)$ & 0.347 \\
\hline 157.5 & 32.9632 & 1.79418 & 5.05879 & 559621 & $-1.72843(07)$ & 0.088 \\
\hline 180 & 68.0906 & 1.87809 & 4.97707 & 924537 & $-3.68669(07)$ & 0.209 \\
\hline
\end{tabular}

atoms. In contrast, for other lighter dopants like $\mathrm{N}_{2} \mathrm{O}$, solvation shells are clearly found $[32,33]$. This could be due to the dynamical correlation induced by the kinetic coupling terms, whose effect is more important for light species. Furthermore

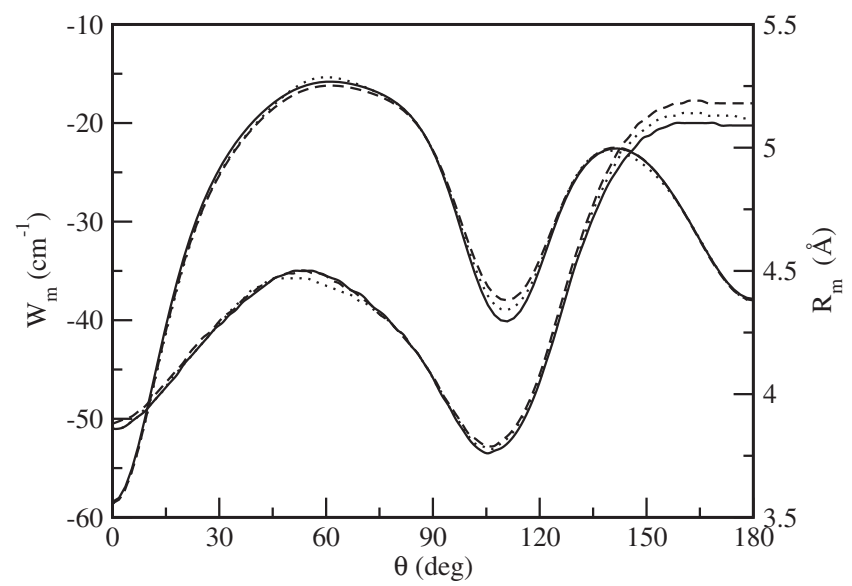

FIG. 1. Minimum energy $W_{m}$ in $\mathrm{cm}^{-1}$, and distance of minimum energy $R_{m}$ in $\AA$, as functions of $\theta$ and $r$. Solid, dotted, and dashed lines correspond to $r=2.271,2.321$, and $2.386 \AA$, respectively. the evaporation energies increase rapidly with cluster size up to $N \sim 14$. Beyond this value we see a reduction in the rate of evaporation. For $N=30$, however, we are still far from the expected asymptotic (bulk) value of $4.94 \mathrm{~cm}^{-1}$ (see, e.g., Ref. [34]).

In Fig. 3(a) we show the angular density distributions for the size range $N=2-30$ normalized to the number of helium atoms. In the inset the distributions obtained from Hartree and variational calculations for $N=2$ are compared: note that they are almost identical, the Hartree being slightly more

TABLE III. Energies (in $\mathrm{cm}^{-1}$ ) of the ICl-He $\mathrm{H}_{N}$ clusters using the Hartree-like scheme. The three columns represent different distributions of the bosons over the orbitals. The values correspond to the lowest-energy " $\Sigma$ " state.

\begin{tabular}{cccc}
\hline \hline$N$ & $M=1$ & $M=2$ & $M=3$ \\
\hline 6 & -86.89 & -85.80 & -85.29 \\
12 & -155.31 & -153.06 & -146.90 \\
18 & -216.49 & -212.67 & -205.89 \\
24 & -274.46 & -267.74 & -262.42 \\
30 & -330.71 & -320.26 & -317.36 \\
\hline \hline
\end{tabular}




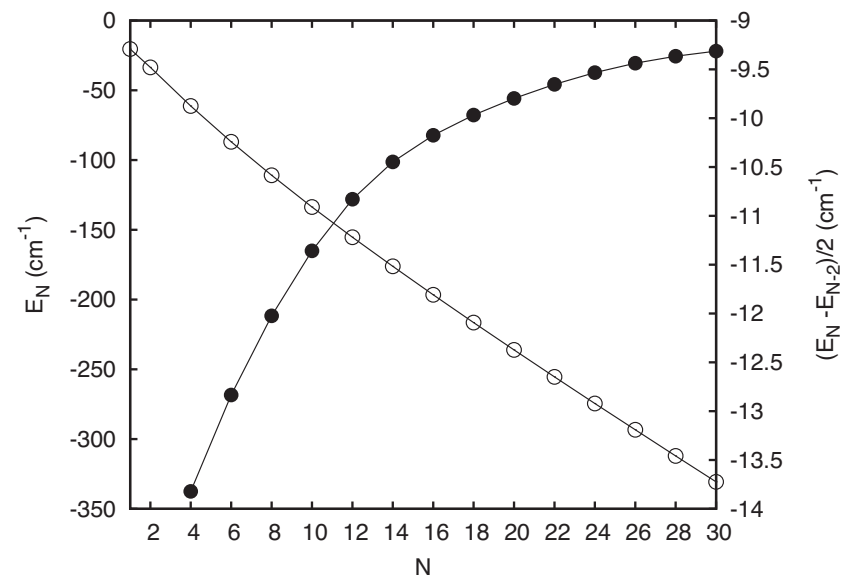

FIG. 2. Total energies $E_{N}$ (open circles) and single-helium evaporation energies, $\left(E_{N}-E_{N-2}\right) / 2$ (solid circles), as a function of the cluster size $N$.

localized. We have also calculated the effective occupation numbers (referred to as $\eta$ ) of the independent-particle (or, equivalently, $\mathrm{He}-\mathrm{ICl}$ ) orbitals. The angular distributions for the five lowest-lying orbitals are shown in Fig. 3(b) while the corresponding energies are displayed in Table IV. The lowest-energy $(n=0)$ orbital is localized in the linear $\mathrm{He}-\mathrm{ICl}$ well, whereas the distributions of the $n=1$ and $n=2$ orbitals are peaking at the asymmetric near T-shaped and antilinear ICl-He wells, respectively [26]. The fourth orbital $(n=3)$ is also rather localized and displays a major peak at $\theta=35^{\circ}$ whereas the fifth orbital $(n=4)$ extends along a broader region and shows peaks around $\theta=90^{\circ}, 120^{\circ}, 145^{\circ}$, and $170^{\circ}$.

The first $\mathrm{He}$ atom populates the axial iodine region (between $0^{\circ}$ and $25^{\circ}$ ) where the global minimum of the $\mathrm{He}-\mathrm{ICl}$ PES is located. For $N=2$, the He-He repulsion causes the density distribution to flow from the linear configuration well to the near T-shaped angular region (between $80^{\circ}$ and $140^{\circ}$ ), and $\eta=0.9$ and 1.1 for $n=0$ and 1, respectively. For $N=4$, the population of the near T-shaped configuration orbital $n=1$ increases to about 2.8 , whereas the the He density at the axial iodine region remains nearly constant. Thus, the variation of $\eta$ for the $n=0$ orbital is only 1.3 for the size range $N$ $=2-30$. In contrast, the population of the $n=1$ orbital increases rapidly with cluster size up to $N \sim 14$ (for which $\eta$ $=8$ ). Beyond this cluster size, it increases at a lower rate and without giving any indication of saturation. Similarly to the $n=0$ orbital, the population of the $n=2$ orbital, which is located in the antilinear well, increases very slowly as the cluster grows in size. For $N=4$, its population is about 0.2 , and a side peak centered around $\theta=170^{\circ}$ appears. For $N=8$, the $n=4$ orbital starts to get populated, a shoulder being appearing at around $\theta \approx 150^{\circ}$. The $\eta$ values of both $n=3$ and $n=4$ orbitals reach a value of about 1 for $N=14$ and, therefore, there is significant nonzero He density between the peaks at the axial iodine region and the central peak centered at the near T-shaped well. For $N=20$, the populations of the $n=3$ and $n=4$ orbitals reach the values of 2 and 1.5 , respectively, and two secondary peaks centered around $\theta=42^{\circ}$ and $78^{\circ}$ are clearly apparent. For larger cluster sizes, the angular density distributions show no additional features and maintain the
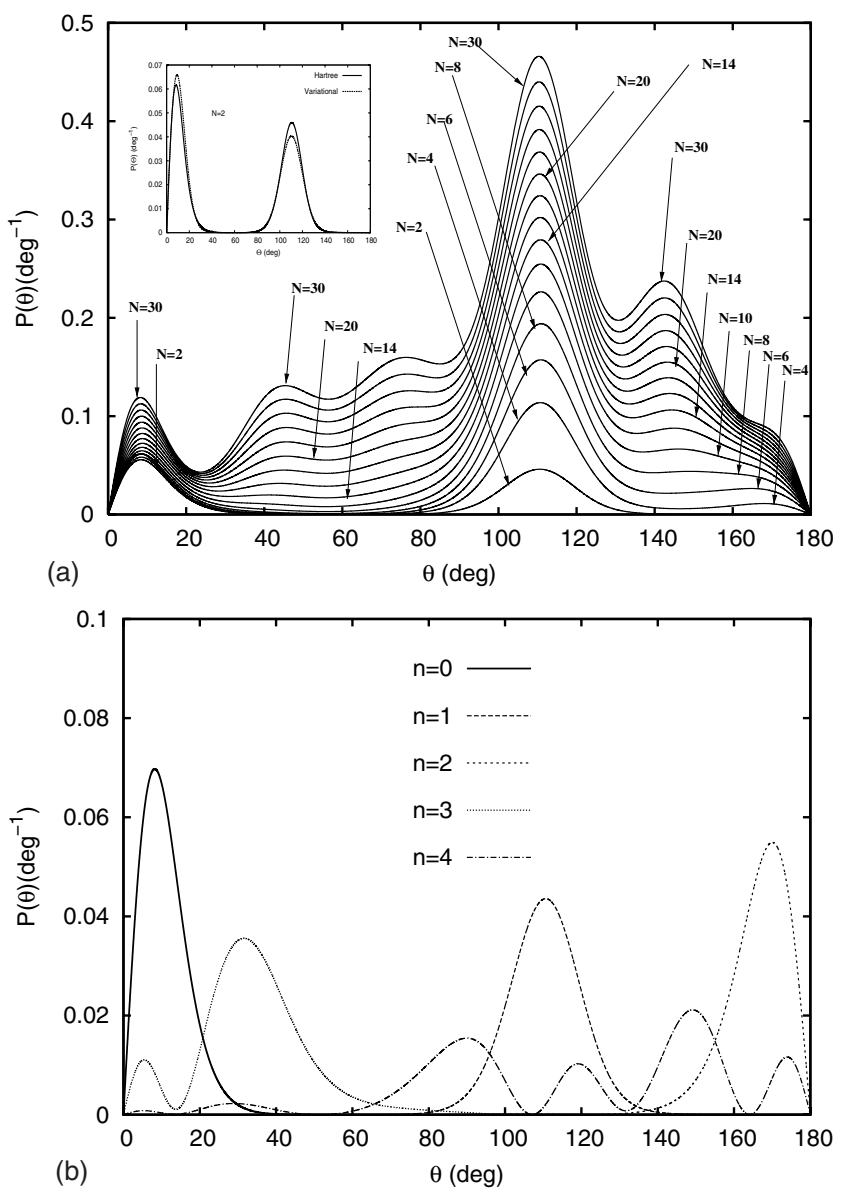

FIG. 3. (a) Computed angular density distributions for the $\mathrm{He}$ atoms surrounding the $\mathrm{ICl}$ molecule for the different cluster sizes. Inset: Comparison of the angular distributions for two helium atoms from variational (full lines) and Hartree calculations (dashed lines). The distributions are normalized to the number of helium atoms, $N$. (b) Angular distributions of the five lowest independent-particle picture orbitals.

sort of five-peak structure of the distribution for $N=20$. In summary, the helium angular distributions show an anisotropic layering around $\mathrm{ICl}$ of the quantum solvation structure, reflecting the strong anisotropy of the $\mathrm{He}-\mathrm{ICl}$ potential energy surface. The most intense feature is the peak centered at the near T-shaped potential minimum. For $N=30$ this peak contains about $14 \mathrm{He}$ atoms axially distributed around the $\mathrm{ICl}$ molecular axis. This angular region is, however, incom-

TABLE IV. Energies (in $\mathrm{cm}^{-1}$ ) of the five lowest independentparticle picture orbitals for the three ICl bond lengths $r=2.271$, 2.321, and $2.386 \AA$.

\begin{tabular}{cccc}
\hline \hline Bound state & $r=2.271$ & $r=2.321$ & $r=2.386$ \\
\hline$n=0$ & -20.24 & -20.38 & -20.68 \\
$n=1$ & -16.57 & -16.14 & -15.80 \\
$n=2$ & -13.56 & -13.49 & -13.68 \\
$n=3$ & -7.49 & -7.61 & -7.86 \\
$n=4$ & -6.67 & -6.78 & -6.75 \\
\hline \hline
\end{tabular}




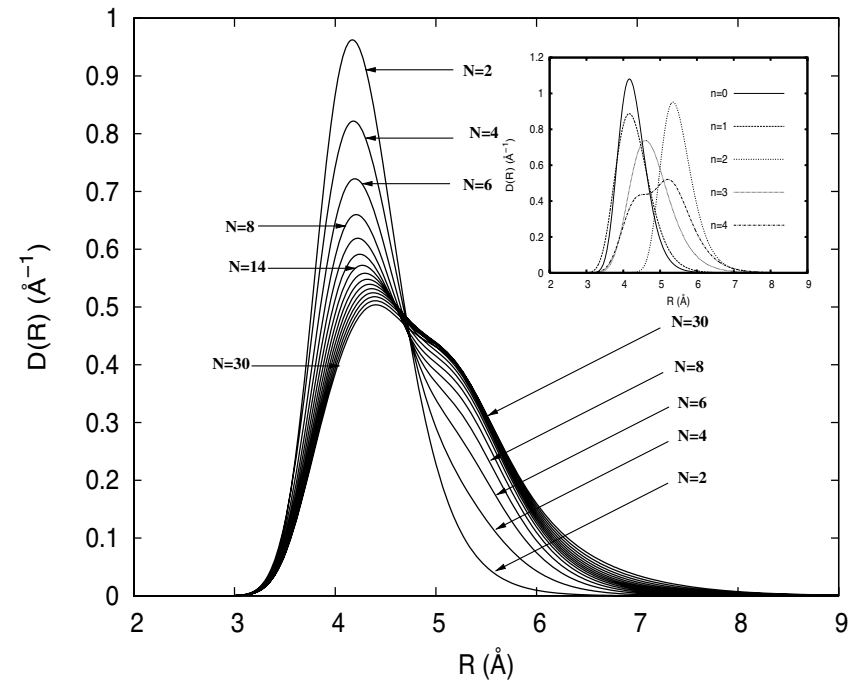

FIG. 4. Radial densities for the helium distributions surrounding the ICl dopant molecule. The distributions are normalized to 1 . Inset: Radial distributions of the five lowest independent-particle picture orbitals.

plete and can still accommodate more helium atoms. The distributions become increasingly more isotropic as the cluster grows in size. However, for the largest cluster size examined here, $N=30$, the angular distribution is still markedly anisotropic and far from the expected isotropic profile in the nanodroplet regime. This is in contrast to the previously studied ${ }^{4} \mathrm{He}-\mathrm{Br}_{2}$ case [19], for which a rather isotropic (and insensitive to the cluster size) distribution is already found for $N=18$, but in agreement with the RQMC calculations by Paolini et al. [35] on ${ }^{4} \mathrm{He}-\mathrm{OCS}$ and ${ }^{4} \mathrm{He}-\mathrm{HCN}$ polar systems, indicating that the rate of approach to the nanodroplet regime is mainly determined by the anisotropy and strength of the specific He-dopant interaction and not by the molecular weight of the dopant.

In Fig. 4, the radial helium density distributions around the dopant's mass center are represented for different cluster sizes. The radial density distributions for the five lowestlying orbitals are also shown in the inset. We see that the distributions of the $n=0$ and $n=1$ orbitals are peaked at $R$ around $4 \AA$. Due to the lighter mass of the $\mathrm{Cl}$ atom, the distribution of the $n=2$ orbital, localized at the antilinear ICl-He well, is clearly peaked at a larger distance to the $\mathrm{ICl}$ mass center of about $5.3 \AA$. Note in Fig. 4 that along the main peak centered at $R$ nearby $4.2 \AA$, a secondary feature centered at $R \sim 5.5 \AA$ appears for $N=8$ (for which the $\eta$ value of the $n=2$ orbital is nearby one). Overall, we see that the two-peak structure of the radial distributions becomes more diffused and more shifted towards larger $R$ distances as the cluster size increases.

We now wish to further consider the dependence of the total energy on the $\mathrm{ICl}(X)$ bond distance. As mentioned, we have examined $\mathrm{ICl}$ bond length distances of $r=2.271,2.321$, and $2.386 \AA$ and found that for distances near the equilibrium bond length the energies can be fitted to better than $2 \%$ accuracy with the logarithmic expression,

$$
E_{N}(r)=A_{N} \log (r-2)+B_{N}
$$

The values of the parameters, along with the corresponding statistical errors, as a function of the cluster size are displayed in Table V. For comparison, the energies of the five lowest independent-boson orbitals are displayed in Table IV for the three $\mathrm{ICl}$ bond length values. One sees in the table that, in contrast to the $n=0$ and 3 cases, the binding energy

TABLE V. Cluster size evolution of different quantities: $A_{N}$ and $B_{N}$ parameters in Eq. (17) together with their statistical errors, averaged values of the squared orbital angular momentum, one half of the VP widths from Eq. (13) (in parentheses, powers of 10), and frequency shift and cross-section value (relative to the $N=10$ size) for the maximum of the corresponding $R(0)$ branch.

\begin{tabular}{ccccccc}
\hline \hline$N$ & $A_{N}\left(\mathrm{~cm}^{-1}\right)$ & $B_{N}\left(\mathrm{~cm}^{-1}\right)$ & $\left\langle\mathbf{L}^{2}\right\rangle($ a.u. $)$ & $\Gamma / 2\left(\mathrm{~cm}^{-1}\right)$ & $\omega\left(\mathrm{cm}^{-1}\right)$ & $\sigma$ \\
\hline 1 & $1.22 \pm 0.02$ & $-21.82 \pm 0.02$ & 17.63 & $1.35(-4)$ & 0.199474 & 10.6068 \\
2 & $1.12 \pm 0.04$ & $-32.41 \pm 0.06$ & 28.44 & $1.36(-4)$ & 0.220891 & 10.3631 \\
4 & $4.54 \pm 0.08$ & $-56.24 \pm 0.10$ & 40.55 & $4.11(-4)$ & 0.254435 & 3.5229 \\
6 & $6.64 \pm 0.09$ & $-79.56 \pm 0.11$ & 48.47 & $8.22(-4)$ & 0.274715 & 1.7661 \\
8 & $8.50 \pm 0.10$ & $-101.50 \pm 0.12$ & 54.34 & $1.16(-3)$ & 0.293495 & 1.2499 \\
10 & $9.95 \pm 0.10$ & $-122.60 \pm 0.13$ & 58.80 & $1.45(-3)$ & 0.308136 & 1.0000 \\
12 & $11.00 \pm 0.13$ & $-143.13 \pm 0.17$ & 61.93 & $1.69(-3)$ & 0.318816 & 0.8664 \\
14 & $11.73 \pm 0.18$ & $-163.31 \pm 0.23$ & 64.59 & $1.90(-3)$ & 0.325956 & 0.7662 \\
16 & $12.32 \pm 0.24$ & $-183.14 \pm 0.31$ & 67.63 & $2.07(-3)$ & 0.331057 & 0.7018 \\
18 & $12.85 \pm 0.31$ & $-202.63 \pm 0.39$ & 71.30 & $2.23(-3)$ & 0.335137 & 0.6511 \\
20 & $13.37 \pm 0.37$ & $-221.79 \pm 0.48$ & 75.61 & $2.38(-3)$ & 0.338617 & 0.6089 \\
22 & $13.91 \pm 0.44$ & $-240.66 \pm 0.57$ & 80.51 & $2.54(-3)$ & 0.341797 & 0.5726 \\
24 & $14.46 \pm 0.51$ & $-259.25 \pm 0.65$ & 86.01 & $2.68(-3)$ & 0.344797 & 0.5409 \\
26 & $15.04 \pm 0.57$ & $-277.61 \pm 0.73$ & 92.11 & $2.83(-3)$ & 0.347797 & 0.5129 \\
28 & $15.66 \pm 0.62$ & $-295.77 \pm 0.80$ & 98.83 & $2.98(-3)$ & 0.350857 & 0.4880 \\
30 & $15.99 \pm 0.65$ & $-304.79 \pm 0.833$ & 106.22 & $3.12(-3)$ & 0.354037 & 0.4657 \\
\hline \hline
\end{tabular}


for the orbital $n=1$ increases as the bond length decreases. This behavior reflects the significant lowering of the near I-shaped well as $r$ decreases (to be compared with the rather weak $r$ dependence of the linear and antilinear wells, see Fig. 1 ), since the $n=1$ orbital is chiefly localized in that potential region. One should also note that the $r$ dependence is more marked for the $n=1$ independent-particle orbital while the $n=4$ orbital, being spatially more extended [see Fig. 3(b)], has an intermediate behavior and it exhibits its energy minimum at the equilibrium ICl bond length. Note now in Table $\mathrm{V}$ that the sign of the "slope" parameter $\mathrm{A}_{N}$ is negative for $N=1$, reflecting the $r$ dependence of the occupied $n=0$ lowest-energy orbital. For $N=2$, the population of $n=1$ orbital is slightly larger (0.2) than the one of the $n=0$ orbital. Therefore, the $r$ dependence of the near I-shaped well dominates and the sign of the slope becomes positive. From $N$ $=2$ to $N \sim 14$, the value of the slope increases quite fast as a consequence of the rapid increase of the $\eta$ value for the $n$ $=1$ orbital. Hereafter, there is a reduction in the rate of slope increase. This is a consequence of the slower rate of population for the near I-shaped peak (see Fig. 3) and the filling of secondary peaks localized at potential regions for which the $r$ dependence is extremely weak. From $N=14$ the deviation from the logarithmic fitting starts to become noticeable.

In Table $\mathrm{V}$ we also list the averaged squares of the total orbital angular momentum as a function of the cluster size. For the smaller clusters $(N \leqslant 8)$, they increase quickly with size, then show an almost linear increase with $N$ values between 10 and 16, and finally show a superlinear growth for the larger clusters up to $N=30$. Together with the $r$-dependent binding energy of the cluster, $\langle\mathbf{L}\rangle^{2}$ contributes to the distortion of the intramolecular potential of the ICl molecule, see Eq. (4), determining the effective potential for the solvated molecule. So, large values of the total orbital angular momentum push the effective equilibrium bond length to longer distances leading to increase the effective moment of inertia.

\section{Spectral simulations}

The IR spectral simulations corresponding to the process,

$$
\mathrm{ICl}\left(X, v_{i}=0\right)+h \nu \rightarrow \operatorname{ICl}\left(X, v_{f}=1\right),
$$

in an environment of $N$ bosonic ${ }^{4} \mathrm{He}$ atoms were carried out assuming a rotational temperature $T=2 \mathrm{~K}$ as in precedent works simulating Raman spectra [17,18]. For the clusters studied here, the particular value of $T$ has only a minor incidence in modifying the relative intensities of the different branches, although due to the $3-j$ symbols appearing at Eq. (12), $R(0)$ always dominates over the rest. A natural broadening of the spectral lines has been included, as described in Sec. II C, by using a simple model which relies on the adiabatic angular treatment of Ref. [30] for the VP process He $-\mathrm{ICl}\left(X, v_{f}=1\right) \rightarrow \mathrm{He}+\mathrm{ICl}\left(X, v_{f}^{\prime}=0\right)$. In Fig. 5 we show the ground state energy of the complex, averaged over the $v=1$ vibrational state of the ICl molecule, and the VP half width as functions of the angle $\theta$. The similarity between the angular dependence of the energy and the angular dependence of the minimum energy path shown in Fig. 1 is evident. The

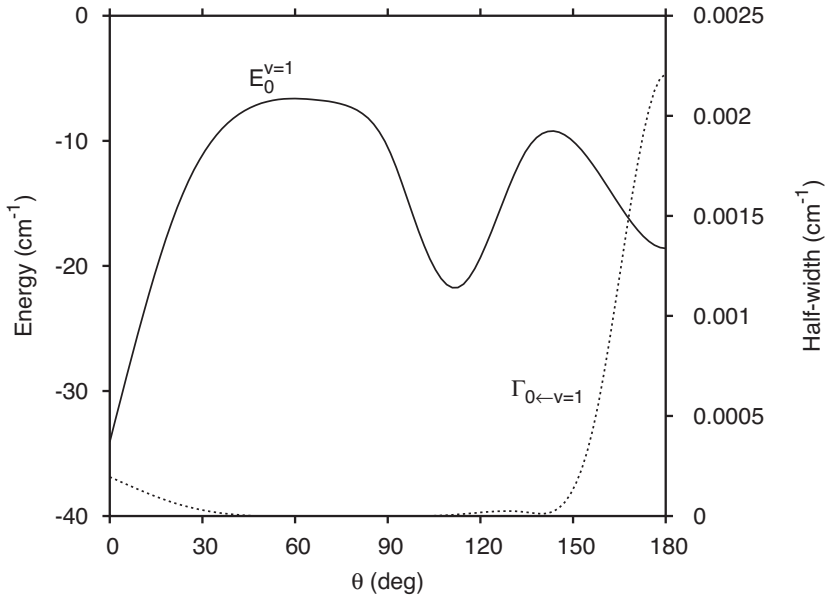

FIG. 5. Computed ground stretching energy and half width for the VP process $\mathrm{He}-\mathrm{ICl}\left(X, v_{f}=1\right) \rightarrow \mathrm{He}+\mathrm{ICl}\left(X, v_{f}^{\prime}=1\right)$, as functions of the orientation $\theta$.

width $\gamma$ is negligible, except in the vicinity of the linear and antilinear arrangements. This function is used together with the distributions shown in Fig. 3(a) as integrands in Eq. (13).

Figure 6 displays the IR profiles of the first four intense branches, $R(0), P(1), R(1)$, and $P(2)$, for the cases $N=2,10$, 20 , and 30 . They are plotted as functions of the frequency shift with respect to the $\left(J_{i}=0, v_{i}=0\right) \rightarrow\left(J_{f}=0, v_{f}=1\right)$ "forbidden" transition ( $\left.380.084899 \mathrm{~cm}^{-1}\right)$ in the bare ICl molecule. One notices the absence of the $Q$ branches, which is consistent with what has been found experimentally for the linear OCS molecule [4]. The profiles display a blueshift, albeit with a decreasing rate, as the size of the cluster increases.

In Table $\mathrm{V}$ we show the cluster size dependence of the VP half width (fifth column), the location (frequency shift) of the maximum photoabsorption cross section (sixth column), and its magnitude relative to the value at $N=10$ (last column)

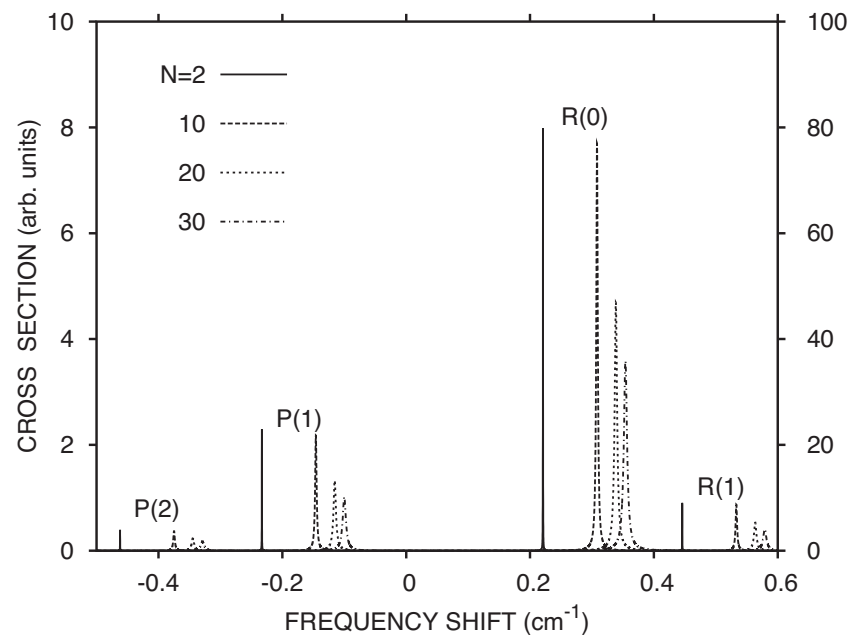

FIG. 6. IR absorption cross sections, for the size selected clusters, showing the first four more intense branches. Cross sections corresponding to $N=2$ are referred to the right vertical axis, while the rest $(\sim 10$ times weaker) are represented on the left one. 
for the dominant $R(0)$ branch. All three quantities exhibit monotonic size dependencies with a convergence tendency. For the cases $N=1$ and 2 the frequencies appear toward the red from the $\left(J_{i}=0, v_{i}=0\right) \rightarrow\left(J_{f}=1, v_{f}=1\right)$ transition $\left(0.226277698 \mathrm{~cm}^{-1}\right)$ of the bare ICl molecule, while for larger clusters the effect of solvation is a shift to the blue. As long as the ICl molecule is only slightly perturbed, its energy levels can be approximated as $E_{J v} \approx E_{v}+B_{v} J(J+1)$, where $B_{v}$ are effective rotational constants depending on the vibrational excitation and the centrifugal distortions are neglected. Hence, size dependent $B_{0}$ and $B_{1}$ values can be obtained from the relative positions of the $R$ and $P$ branches. For instance, denoting by $\Delta_{10}$ the distance in frequency between the $R(1)$ and $R(0)$ lines, and by $\Delta_{21}$ the corresponding magnitude involving $R(2)$ and $R(1)$, one readily obtains $B_{0}$ $=1.5 \Delta_{10}-\Delta_{21}$ and $B_{1}=\Delta_{10}-0.5 \Delta_{21}$. In this way, a tiny increase of the rotational constants as the cluster size grows is found. In wave numbers, $B_{0}$ ranges from 0.113806 at $N=1$ to 0.113846 at $N=30$. The corresponding values for $B_{1}$ are 0.113134 and 0.113173 . For the bare ICl molecule these rotational constants become $B_{0}=0.113813$ and $B_{1}$ $=0.113139 \mathrm{~cm}^{-1}$, which are lower than those found for all sizes except $N=1$. One can interpret these results as an indication of an effective increase in the moment of inertia of ICl when it is solvated by one He atom, and an effective decrease in the moment of inertia when it is solvated in a larger cluster. Since rather moderate values of the square of the orbital angular momentum are attained, the behavior of the moment of inertia is a direct consequence of the dependence of the binding energy on the ICl bond length, which from $N=2$ decreases as $r$ increases. In fact, for $N$ from 4 to 30, the minimum of the effective potential for the solvated ICl is located at shorter $r$ values than the minimum of the potential of the isolated molecule, giving rise to a reduction in the moment of inertia. This is in apparent contradiction with the experimental findings in droplets [4]. However, for much larger cluster sizes than those studied here, note that since the binding energy should become insensitive to variations of $r$, and $\left\langle\mathbf{L}^{2}\right\rangle$ could attain large values, one expects that the equilibrium bond length of the solvated molecule be larger then the corresponding to the isolated molecule, recovering the agreement with the experiment.

\section{SUMMARY AND OUTLOOK}

Using a realistic potential, which is based on accurate $a b$ initio calculations, we performed a Hartree-type study of the energies, density distributions, and IR spectra of $\operatorname{ICl}(\mathrm{X})$ $-\left({ }^{4} \mathrm{He}\right)_{N}$ clusters in the size range of $N=1-30$. This study extends our earlier application of the method [19], which is in essence a "multiconfigurational" treatment, to polar molecules embedded in a finite-size bosonic solvent. Our main findings can be summarized as follows:

(1) The $\Sigma(\Lambda=0)$ state of the clusters, when all the $\mathrm{He}$ atoms occupy the same orbital, is the energetically most preferred one.

(2) The helium density distributions around the ICl molecule are strongly anisotropic, which is a consequence of the anisotropy of the $\mathrm{He}-\mathrm{ICl}$ potential; the anisotropy of the distribution is quite apparent even for the largest cluster considered here $(N=30)$.

(3) The size variations of the cluster energies and densities are smooth with no indication of shell effects.

(4) The variation of the cluster energies with the $\mathrm{ICl}$ bond length is well represented by a logarithmic function. The "slope" of the logarithmic dependence increases with the cluster size. The rate of increase is substantial up to $N=14$, but slows down thereafter.

(5) The calculated IR spectra of the ICl molecule embedded in an environment of ${ }^{4} \mathrm{He}$ atoms lack the Q branches as long as only $\Sigma$ states are involved.

(6) For most clusters in the size range considered here the effective momement of inertia of the solvated ICl molecule is smaller than that of the bare ICl. It is expected, however, that the opposite will become true as the number of solvent ${ }^{4} \mathrm{He}$ atoms becomes large enough.

Our future studies will explore the cases of ICl embedded in ${ }^{3} \mathrm{He}$ and mixed ${ }^{3} \mathrm{He} /{ }^{4} \mathrm{He}$ clusters and will address the issue of the role played by the nuclear spin statistics in the phenomenon of finite-size quantum solvation. We will also consider clusters of para $-\mathrm{H}_{2}$ and ortho $-\mathrm{H}_{2}$, as well as their mixtures, as solvation environments. One may expect these to produce effects similar to those found in bosonic and fermionic helium clusters. Data obtained in recent spectroscopic studies of hydrogen clusters [36] and of different molecules embedded in them [37-39] provide both an impetus and a calibration basis for theory.

\section{ACKNOWLEDGMENTS}

This work has been partially supported by the DGICYT Spanish Grant No. FIS2004-02461 and the European Project No. R113-2003-506079. M.P.deL.-C. was supported by the "Ramón and Cajal" Programme and J.J. by the Office of Basic Energy Sciences, Division of Chemical Sciences, Geosciences, and Biosciences, U.S. Department of Energy under Contract No. DE-AC-02-06CH11357. The calculations presented here were performed at the Instituto de Matemáticas y Física Fundamental (CSIC), at CINECA (the SuperComputer Center of the University of Bologna), and CESGA (the SuperComputer Center of Galicia).
[1] J. P. Toennies and A. F. Vilesov, Angew. Chem., Int. Ed. 43, 2622 (2004).

[2] S. Goyal, D. L. Schutt, and G. Scoles, Phys. Rev. Lett. 69, 933 (1992).
[3] M. Hartmann, R. E. Miller, J. Toennies, and A. F. Vilesov, Phys. Rev. Lett. 75, 1566 (1995).

[4] S. Grebenev, J. P. Toennies, and A. F. Vilesov, Science 279, 2083 (1998). 
[5] S. Grebenev, M. Hartmann, M. Havenith, B. Sartakov, J. P. Toennies, and A. F. Vilesov, J. Chem. Phys. 112, 4485 (2000).

[6] K. Nauta and R. E. Miller, J. Chem. Phys. 115, 10138 (2001).

[7] K. Nauta and R. E. Miller, J. Chem. Phys. 117, 4846 (2002).

[8] C. M. Lindsay and R. E. Miller, J. Chem. Phys. 122, 104306 (2005).

[9] J. Tang and A. R. W. McKellar, J. Chem. Phys. 119, 754 (2003).

[10] A. R. W. McKellar, J. Chem. Phys. 121, 6868 (2004).

[11] For a recent review, see M. Barranco, R. Guardiola, S. Hernández, R. Mayol, J. Navarro, and M. Pí, J. Low Temp. Phys. 142, 1 (2006).

[12] S. Baroni and S. Moroni, in Quantum Monte Carlo Methods in Physics and Chemistry, edited by P. Nightingale and C. J. Umrigar, NATO Series, Mathematical and Physical Sciences, Vol. 525 (Kluwer Academic, Boston, 1999).

[13] Z. E. Zillich, F. Paesani, Y. Kwon, and K. B. Whaley, J. Chem. Phys. 123, 114301 (2005).

[14] D. Blume, M. Lewerenz, P. Niyaz, and K. B. Whaley, Phys. Rev. E 55, 3664 (1997).

[15] F. Paesani, A. Viel, F. A. Gianturco, and K. B. Whaley, Phys. Rev. Lett. 90, 073401 (2003).

[16] S. Moroni, A. Sarsa, S. Fantoni, K. E. Schmidt, and S. Baroni, Phys. Rev. Lett. 90, 143401 (2003).

[17] D. López-Durán, M. P. de Lara-Castells, G. Delgado-Barrio, P. Villarreal, C. D. Paola, F. A. Gianturco, and J. Jellinek, Phys. Rev. Lett. 93, 053401 (2004).

[18] D. López-Durán, M. P. de Lara-Castells, G. Delgado-Barrio, P. Villarreal, C. D. Paola, F. A. Gianturco, and J. Jellinek, J. Chem. Phys. 121, 2975 (2004).

[19] M. P. de Lara-Castells, D. López-Durán, G. Delgado-Barrio, P. Villarreal, C. D. Paola, F. A. Gianturco, and J. Jellinek, Phys. Rev. A 71, 033203 (2005).

[20] A. Valdés, R. Prosmiti, P. Villarreal, and G. Delgado-Barrio, J. Chem. Phys. 122, 044305 (2005).

[21] R. Prosmiti, C. Cunha, P. Villarreal, and G. Delgado-Barrio, J. Chem. Phys. 116, 9249 (2002).

[22] R. A. Aziz and M. J. Slaman, J. Chem. Phys. 94, 8047 (1991).
[23] C. D. Paola, F. A. Gianturco, D. López-Durán, M. P. de LaraCastells, G. Delgado-Barrio, P. Villarreal, and J. Jellinek, ChemPhysChem 6, 1348 (2005).

[24] A. Valdés, R. Prosmiti, P. Villarreal, and G. Delgado-Barrio, Mol. Phys. 102, 2277 (2004).

[25] T. González-Lezana, M. I. Hernández, G. Delgado-Barrio, A. A. Buchachenko, and P. Villarreal, J. Chem. Phys. 105, 7454 (1996).

[26] R. Prosmiti, C. Cunha, P. Villarreal, and G. Delgado-Barrio, J. Chem. Phys. 117, 7017 (2002).

[27] A. Valdés, R. Prosmiti, P. Villarreal, and G. Delgado-Barrio, J. Chem. Phys. 125, 014313 (2006).

[28] P. Villarreal, O. Roncero, and G. Delgado-Barrio, J. Chem. Phys. 101, 2217 (1994).

[29] J. Fernández-Rico, in Self-Consistent Field, Theory and Applications, edited by R. Carbo and M. Klobukowski, Physical and Theoretical Chemistry, Vol. 70 (Elsevier, New York 1990).

[30] J. A. Beswick and G. Delgado-Barrio, J. Chem. Phys. 73, 3653 (1980).

[31] K. P. Huber and G. Herzberg, Constants of Diatomic Molecules (Van Nostrand Reinhold, New York, 1979).

[32] S. Moroni, N. Blinov, and P. N. Roy, J. Chem. Phys. 121, 3577 (2004).

[33] Y. Xu, N. Blinov, W. Jäger, and P. N. Roy, J. Chem. Phys. 124, 081101 (2006).

[34] M. H. Kalos, M. A. Lee, P. A. Whitlock, and G. V. Chester, Phys. Rev. B 24, 115 (1981).

[35] S. Paolini, S. Fantoni, S. Moroni, and S. Baroni, J. Chem. Phys. 123, 114306 (2005).

[36] G. Tejada, J. M. Fernández, S. Montero, D. Blume, and J. P. Toennies, Phys. Rev. Lett. 92, 223401 (2004).

[37] S. Grebenev, B. Sartakov, J. P. Toennies, and A. F. Vilesov, Science 289, 1532 (2000).

[38] J. Tang and A. R. W. McKellar, J. Chem. Phys. 121, 3087 (2004).

[39] S. Moroni, M. Botti, S. De Palo, and A. R. W. McKellar, J. Chem. Phys. 122, 094314 (2005). 